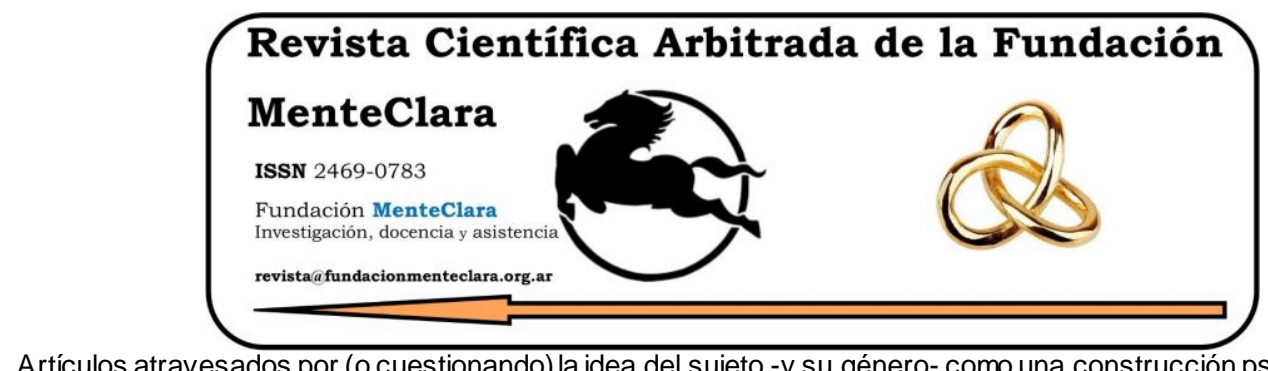

Artículos atravesados por (o cuestionando) la idea del sujeto -y su género- como una construcción psicobiológica de la cultura. Articles driven by (or questioning) the idea of the subject -and their gender- as a cultural psychobiological construction.

Vol. 7 (2022), enero-diciembre ISSN 2469-0783

https: / / datahub.io/ dataset/2022-7-e260

\title{
ESTUDIO DEL OPTIMISMO Y LA AUTOESTIMA RELACIONADO AL USO DE LAS REDES SOCIALES EN JÓVENES ADOLESCENTES
}

\author{
OPTIMISM AND SELF-ESTEEM STUDY ON THE USE OF SOCIAL MEDIA AMONGST \\ YOUNG TEENAGERS
}

Carlos José Luna jose.1una@uflo.edu.ar

Universidad de Flores, Facultad de Psicología y Ciencias Sociales, Argentina.

Cómo citar este artículo / Citation: Luna, C. (2022). Estudio del optimismo y la autoestima relacionado al uso de las re des sociales en jóvenes adole scentes. Revista Cientifica Arbitrada de la Fundación MenteClara, Vol. 7 (260). DOI:

https://doi.org/ 10.32351/rca.v7.260

Copyright: (C) 2022 RCAFMC. Este artículo de acce so abierto es distribuido bajo los términos de la licencia Creative Commons Attribution 4.0 International License (CC BY 4.0). Recibido: 18/01/2022. Aceptado: 04/02/2022 Publicación online: 11/02/2022

Conflicto de intereses: Ninguno que declarar.

\section{Resumen}

En los años recientes las redes sociales han tenido un gran impacto en la formación de los adolescentes y en su bienestar emocional. La psicología estudia los conceptos de la autoestima y el optimismo, los cuales son factores clave en la búsqueda de mejorar el bienestar o mantener un buen nivel del mismo. Es por ello que esta investigación va a indagar cómo se encuentran el optimismo y la autoestima relacionados en el proceso de formación cognitiva del adolescente abordando el impacto en ambas variables y cómo influyen las redes sociales, el cual es el objetivo. A partir de lo expuesto se concluye que el optimismo es una gran herramienta que puede ser usada en el momento que ocurra algún evento negativo que afecte directamente sobre la autoestima y el autoconcepto; lo que se pretende es aislarlos o hacerlos menos recurrentes. Debido a que las redes sociales evolucionan tan rápidamente se sugiere mantenerse actualizado en este ámbito para poder contrarrestar los efectos negativos de vivir en una era digital. 


\begin{abstract}
In recent years, social media have had a great impact on the conformation of teenagers and their emotional well-being. Psychology studies the concepts of selfesteem and optimism, which are key factors in the search to improve well-being or maintain a good level of it. That is why this research will investigate how optimism and self-esteem are correlated in the teenagers' cognitive conformation process, addressing the impact on both variables and how social media influence these variables, which is the objective. Based on the above, it is concluded that optimism is a great tool that can be used when a negative event occurs that directly affects selfesteem and self-concept; the aim is to isolate them or make them less recurrent. Because social networks evolve so rapidly it is suggested to stay updated in this area to be able to counteract the negative effects of living in a digital age.
\end{abstract}

Palabras Claves: Optimismo; autoestima; redes sociales; bienestar emocional; relaciones sociales

Keyw ords: Optimism; self-esteem; social networks; emotional well-being; social relationships 


\section{Introducción}

Actualmente las redes sociales ayudan a mantenerse conectados y estar más cerca de otros. En el contexto actual, pasando por una pandemia que afectó mundialmente en diversos aspectos, tanto económicos y sociales así como en los políticos y culturales, las redes fueron un aliado para poder estar en contacto con el resto del mundo mientras concluía el aislamiento. Ahora bien, su uso en exceso puede traer consecuencias negativas que son mayormente asociadas a la autoestima. En los jóvenes adolescentes de la época es más notorio el impacto negativo de las redes.

El optimismo, en una medida justa, ayuda a mantener niveles adecuados de expectativas y da una mejor visión sobre la vida en general, este toma importancia cuando queremos cuidar el nivel de la autoes tima, aquel factor importante en la felicidad y el bienestar emocional.

Se toma a la población adolescente para el estudio ya que son los más afectados de forma negativa, por todo lo que conlleva el uso de las redes sociales, la forma en la que se relacionan con otras personas y las conductas tomadas cuando los niveles de autoestima son bajos en el individuo. A grandes rasgos, se intenta observar la forma en la que afecta la adicción al smartphone en las relaciones interpersonales, en cómo el adolescente existe la tendencia a presentar un mal autoconcepto.

\section{Autoestima}

$\mathrm{El}$ autoconcepto tiene que ver con las creencias que tiene el individuo sobre sí mismo, pudiendo también llamarse autopercepción. La 
autoestima tiene que ver con el juicio de valor que le damos a esa autopercepción, si es bueno o es malo, es una evaluación (Fagundes, Marot, \& Natividade, 2021). La autoestima es la visión que tiene la persona sobre sí misma, puede ser negativa o positiva. Algunos de los factores que se encuentran a atados o asociados a niveles altos de autoestima son la adaptación social, la felicidad y una menor vulnerabilidad a presentar conductas de riesgo.

La contraparte de esto es una valoración negativa de la vida, dificultad para adaptarse a situaciones incómodas o menos afortunadas, también un mayor riesgo de conductas negativas que ponen en descuido la salud integral del individuo, todo esto es observable en un bajo nivel de autoestima (González, Mesa, Zayas, \& Molinero, 2018). Si bien no existe una única definición de la autoestima, la misma ha sido estudiada como un constructo que es un indicador clave en la salud mental y tiene un gran interés en el área clínica de la psicología.

Para el individuo la autoestima no toma como referencia la mirada de los demás sino la forma en que la persona siente que los demás lo observan y la misma se desarrolla desde que el individuo comienza a tomar conciencia de sí mismo (León Gualda \& Lacunza, 2020). El primer ambiente en el que se va desarrollando este aspecto evaluativo es en el entorno familiar y las valoraciones de los familiares tienen una gran influencia en cómo se va creando esta percepción de sí mismo, por medio de ciertos valores se le va enseñando e inculcando cómo debería ser, los aspectos y formas deseables. El contexto académico también es factor clave en la formación de la autoestima. 
Existe un vínculo entre las relaciones sociales y la autoestima ya que ella puede impactar en la forma en la que percibimos la relación que tenemos con otros, de cierta forma proyectar creencias internas en esa perspectiva que se tiene de la relación con otra persona (Harris \& Orth, 2020). Los jóvenes en edad universitaria son generalmente más propensos a generar ciertas adicciones. Una de ellas es la adicción al smartphone, y en esta adicción, la principal variable afectada es la autoestima.

Una baja autoestima generalmente impulsa o funciona como desencadenante de esta adicción, las redes sociales juegan un rol importante en la sociedad actual, las personas suelen usarlas como un medidor para la aprobación social, por medio de los likes, comentarios o el alcance de las publicaciones. Hay que considerar que la autopercepción también tiene un gran papel en la forma en la que un individuo se presenta, por ejemplo, en Instagram y en muchos casos el hecho de no saber manejar o controlar esa constante comparación con otra persona, puede conllevar a ciertas actitudes no muy sanas, como las de idealizar ciertos rasgos o cierto estatus social y querer de alguna forma recrearlo, afectando negativamente en la autoestima (Romero Rodríguez \& Aznar Díaz, 2019).

Aunque esa comparativa mayormente es asociada a lo que está mal, la misma puede llegar a ser positiva, motivando al individuo a mejorar en ciertos aspectos. La comparación social es tanto positiva como negativa en este aspecto (Fagundes, Marot, \& Natividade, 2021). Las relaciones sociales pueden ser medidas por las habilidades: aquellas aptitudes o técnicas necesarias para llevar a cabo de forma competente una tarea 
interpersonal, todo esto es un comportamiento adquirido o aprendido, y no un rasgo de la personalidad en sí. Mientras más alta sea la autoestima del individuo a lo largo de su proceso de crecimiento, más asertivo será en la forma en la que se relaciona con los demás (León Gualda \& Lacunza, 2020).

\section{Optimismo}

El optimismo es aquello que se observa de forma continua y estable con respecto a la creencia de que los eventos acontecidos siempre van a ser positivos, también es la destreza de tomar las experiencias vividas desde el mejor punto de vista, esto siempre es tomado desde la realidad y lo posible, sin opción a salir de estas dos variables (García, Díaz, Rodríguez, \& Vallerino, 2018).

Numerosas investigaciones lo definen de distinta forma, no obstante dos ramas del optimismo toman las riendas como enfoques teóricos, uno explicativo y otro disposicional. De acuerdo con el estilo explicativo optimista: la permanencia inestable, la especificidad amplia y la causalidad externa están relacionadas a los eventos negativos. Este estilo explicativo es más fácil de encontrar asociado a una mejor salud, tanto física como psíquica y a una menor probabilidad de padecer enfermedades físicas. El estilo explicativo pesimista sería su contraparte y está relacionado a un empeoramiento en la salud y mayor disposición a enfermedades. El optimismo disposicional está más dirigido hacia lo cognitivo y sobre cómo tener expectativas más elevadas respecto a lo que está próximo a suceder se relaciona con una alta motivación y mayor esfuerzo en ciertas tareas. Este optimismo se encuentra relacionado 
positivamente a una mayor autoestima y de forma negativa hacia los sintomas de un cuadro clínico depresivo (Ferrer, 2020).

Se ha estudiado la posibilidad de que el optimismo no sea unidireccional sino que más bien se trate de dos contrapuestos, los cuales serían optimismo y pesimismo. Es considerado que ambos factores comienzan a ser independientes a medida que el individuo va alcanzandola madurez, de la mano con un proceso meta-cognitivo y de una forma más bien adaptativa pueden ser usadas las perspectivas pesimista u optimista dependiendo de la situación a la cual se tenga que enfrentar (Pérez, et al., 2021)

Existen investigaciones que evidencian que el optimismo irracional, aquel que va más allá de la realidad y de las propias posibilidades, puede impactar tanto positiva como negativamente. Cuando las expectativas no son cumplidas en la forma pensada, una gran cantidad de emociones negativas pueden aparecer. A grandes rasgos, esta creencia reduce el nivel de emociones positivas que resultan o son consecuentes a las experiencias, en la cual una alta expectativa no permite que exista el mismo placer que si hubiese existido una creencia contraria u opuesta (De Meza \& Dawson, 2021).

\section{Autoestima y optimismo}

Los resultados del estudio de la integración de ambas variables encontraron una relación moderada/alta entre el optimismo y la autoestima en el bienestar psicológico. El optimismo es un factor clave en la felicidad y la autoestima por su parte tiene mayor relación con el bienestar psicológico, siendo el autoconcepto el más afectado, positiva y 
negativamente según el nivel de autoestima presentado. Hasta el 55\% de la felicidad puede ser basado en estas dos variables (Moreno \& Marrero, 2015). En los jóvenes es común pasar más de tres horas con el teléfono, conducta que puede llevar a generar una adicción en dicha población (Romero Rodríguez \& Aznar Díaz, 2019).

En las investigaciones sobre la estabilidad de la autoestima a lo largo de la vida se da la posibilidad de que mientras el individuo crece y llega a un mayor estado de madurez, las metas y los logros que se han cumplido sirven como medidor y crítico del autoconcepto (Trzesniewski, Donnellan, \& Robins, 2003).

Hay un impacto en el bienestar y en la salud mental de los jóvenes debido al creciente uso de las redes sociales, cierta tendencia o conducta puede convertirse en un problema cuandollega a generarse una adicción. Aunque algunos aspectos podrían incluso llegar a parecer narcisistas, esto toma la forma de una norma social, en el posteo diario de fotos como los selfies. Las personas suelen compararse con otros como una forma de evaluación de sus habilidades y opiniones propias, esto según una teoria que habla de la comparación social. La adolescencia suele ser un período de formación de la personalidad y en esta etapa puede ser complejo encontrar un punto de autorregulación de varios aspectos. Existe un efecto adverso encontrado en esta presión por llegar a ser como otros (Keles, McCrae, \& Grealish, 2020).

Otro factor a considerar en la forma en la que la autoestima se ve afectada por el uso de las redes sociales es la retroalimentación, los comentarios y las respuestas a las publicaciones pueden ser tomadas de 
manera negativa por el individuo, de forma más bien subjetiva dependiendo del autoconcepto que se tenga (Appel, Gerlach, \& Crusius, 2016).

Podemos destacar la autoestima por encima del optimismo cuando los relacionamos con la forma en la que el uso de las redes sociales impacta en ambas variables. Estudios actuales proveen las bases para varias teorias sobre la autoestima y todas ellas resaltan el importante rol que cumple el hecho de reafirmar las relaciones sociales positivas y la aceptación social, esto es importante debido a que anteriormente era inconsistente el patrón de los resultados en teorias anteriores (Harris $\&$ Orth, 2020).

\section{Conclusiones}

La complejidad de vivir en un contexto en el que las redes sociales toman gran importancia para mantenernos comunicados se hace notar cuando los adolescentes intentan tener cierto estándar impuesto por la presión social que genera ser parte de esto. Tratar de ser como el otro genera un malestar en el individuo, comienza a tener pensamientos negativos sobre su imagen, sus gustos, sus pensamientos, sus ideas e incluso su personalidad. Es importante tener en cuenta que la adolescencia es un momento de la vida en el que la persona no está del todo formada, ni psíquicamente ni cognitivamente y esto la hace más influenciable, los contenidos que consuma en las redes van a ir moldeando sus ideas y el autoconcepto.

Ser optimistas, se encontró en varios estudios e investigaciones, ayuda a tener una base más grande para poder contrarrestar los efectos 
negativos que pueden llegar a presentarse emocionalmente en el individuo (Pérez, et al., 2021). El optimismo genera una confianza en los eventos que irán sucediendo, se enfoca en lo positivo y aparta lo negativo (Moreno \& Marrero, 2015). En los adolescentes con autoestima baja, causada por una adicción al smartphone y las redes, es ideal introducirlos en el concepto de optimismo, así como también los principios básicos de las investigaciones y cómo mejorar sus niveles de autoestima, permitiendo así mejorar su bienestar emocional.

Se sugiere que las investigaciones pongan mayor énfasis en los patrones de conducta encontrados en la adicción a las redes, al ser una era digital, luego de atravesar una pandemia que sumergió a la humanidad casi por completo a lo virtual, se vuelve un tema importante de tratar. Comenzar investigaciones y estudios sobre cómo esto ha afectado y seguirá afectando a las siguientes generaciones que nacieron y crecieron en pandemia.

Es importante que los adolescentes también conozcan sus limitaciones y que sean conscientes de cómo les afecta negativamente las redes sociales y cómo la presión social puede dar el pie para que su bienestar emocional se vea afectado por medio de la autoestima. También se considera mejorar la calidad de vida de la población introduciéndolos a perspectivas optimistas que les sirvan como herramienta para poder evitar las repercusiones negativas de todo lo que conlleva estar dentro de las redes sociales en la actualidad. 


\section{Referencias}

Appe1, H., Gerlach, A., \& Crusius, J. (2016). The interplay between Facebook use, social comparison, envy and de pression. Current opinion in psychology, 9, 44-49.

https://doi.org/10.1016/j.copsyc.2015.10.006

De Meza, D., \& Dawson, C. (2021). Neither an optimist nor a pessimist Be: mistaken expectations lower well-being. Personality and Social Psychology Bulletin, 47(4), 540-550. https://doi.org/10.1177/0146167220934577

Fagundes, L. S., Marot, T. A., \& Natividade, J. C. (2021). Use of Instagram, Social Comparison, and Personality as Predictors of Self-Esteem. Psico-USF, 25, 711-724. https://doi.org/10.1590/1413/82712020250410

Ferrer, C. (2020). El optimismo y su relación con el bienestar psicológico. Revista Científica Arbitrada de la Fundación MenteClara, 5(199). https://doi.org/10.32351/rca.v5.199

García, C., Díaz, M., Rodríguez, A. M., \& Vallerino, V. T. (2018). El optimismo como contribución a la resiliencia de portiva. Revista iberoamericana de psicología del ejercicio y el de porte, 13(1), 131-136. https://dialnet.unirioja.es/servlet/articulo?codigo=6195001

González, P. R., Mesa, Y. M., Zayas, A., \& Molinero, R. G. (2018). Relación entre la autoestima y la satisfación con la visa en una muestra de estudiantes universitarios. Inter national Journal of Developmental and Educational Psychology, 2(1), 67-76.

https:/ / www.re dalyc.org/ articulo.oa?id=349856003007

Harris, M. A., \& Orth, U. (2020). The link between self-esteem and social relationships: A metaanalysis of longitudinal studies. Journal of personality and social psychology, 119(6), 14591477. https:// doi.org/10.1037/ps pp0000265

Keles, B., McCrae, N., \& Grealish, A. (2020). A systematic review: the influence of social media on depression, anxiety and psychological distress in adolescents. International Journal of Adolescence and Youth, 25(1), 79-93. https://doi.org/10.7759/cureus.8627

León Gualda, G., \& Lacunza, A. B. (2020). Autoestima y habilidades sociales en niños y niñas de1 Gran San Miguel de Tucumán, Argentina. Revista Argentina de Salud Pública, 11(42), 22-31. https://www.scielo.org.ar/scieloOrg/php/articleXML.php? pid=S1853810X20200001000228*lang=es

Moreno, Y., \& Marrero, R. J. (2015). Optimismo y autoestima como predictores de bienestar personal: diferencias de género. Revista Mexicana de Psicología, 32(1), 27-36. https: / / www.re dalyc.org/ articulo.oa?id=243045363004

Pérez, S. P., Costa, M. A., Moreno, A. D., López, I. C., López, P. M., Zamorano, M. A., \& Rubio, M. J. (2021). Optimismo disposicional y estrés: claves para promover el bienestar psicológico. Papeles del Psicólogo, 42(2), 135-142. https://doi.org/10.23923/pap.psicol2021.2953

Romero Rodríguez, J. M., \& Aznar Díaz, I. (2019). Análisis de la adicción al smartphone en estudiantes universitarios. Factore s influyentes y correlación con la autoe stima. RED. Revista de Educación a Distancia, 19(60), 6-0. https://doi.org/10.6018/red/60/08

Trzesniewski, K. H., Donnellan, M. B., \& Robins, R. W. (2003). Stability of self-esteem across the life span. Journal of personality and social psychology, 84(1), 205-220.

https://doi.org/10.1037//0022-3514.84.1.205 